\title{
Geometrodynamical formulation of two-dimensional dilaton gravity
}

\author{
Marco Cavagliä* \\ Max-Planck-Institut für Gravitationsphysik, Albert-Einstein-Institut, Schlaatzweg 1, D-14473 Potsdam, Germany
}

(Received 31 July 1998; published 12 March 1999)

\begin{abstract}
Two-dimensional matterless dilaton gravity with an arbitrary dilatonic potential can be discussed in a unitary way, both in the Lagrangian and canonical frameworks, by introducing suitable field redefinitions. The new fields are directly related to the original spacetime geometry and in the canonical picture they generalize the well-known geometrodynamical variables used in the discussion of the Schwarzschild black hole. So the model can be quantized using the techniques developed for the latter case. The resulting quantum theory exhibits the Birkhoff theorem at the quantum level. [S0556-2821(99)01708-7]
\end{abstract}

PACS number(s): 04.60.Ds, 04.20.Fy, 04.60.Kz

\section{INTRODUCTION}

Recently, a lot of attention has been devoted to the investigation of lower-dimensional gravity [1]. The interest in dimensionally reduced theories of gravity relies essentially on their connection to string theory, black hole physics, and gravitational collapse. In this context, two-dimensional models of dilaton gravity play a very important role because of their relation to higher-dimensional gravity and integrable systems.

Two-dimensional dilaton gravity is described by the action [2]

$$
\begin{aligned}
S_{D G M}= & \int_{\Sigma} d^{2} x \sqrt{-\gamma}\left[\mathcal{U}(\phi) \mathcal{R}^{(2)}(\gamma)+\mathcal{V}(\phi)+\mathcal{W}(\phi)(\nabla \phi)^{2}\right] \\
& +S_{M}\left[\phi, \gamma_{\mu \nu}, f_{i}\right],
\end{aligned}
$$

where $\mathcal{U}, \mathcal{V}$, and $\mathcal{W}$ are arbitrary functions of the dilaton, $\mathcal{R}^{(2)}$ is the two-dimensional Ricci scalar, and $S_{M}$ represents the contribution of matter fields $f_{i}$ which include any field but the dilaton $\phi$ and the graviton $\gamma_{\mu \nu}$.

Most of the models studied in some detail in the literature are special cases of the model described by Eq. (1) where dilaton gravity is coupled to scalar, gauge, and fermion fields. (See, for instance, Refs. [3-13] and references therein.) For a given $S_{M}$, Eq. (1) describes a family of models whose elements are identified by the choice of the dilatonic potential. Indeed, classically we may always choose $\mathcal{U}(\phi)=\phi$ and locally set $\mathcal{W}(\phi)=0$ by a Weyl rescaling of the metric [2]. (In this paper we will always make this choice for simplicity [14].) So the matterless sector of Eq. (1) reads [2]

$$
S_{D G}=\int_{\Sigma} d^{2} x \sqrt{-g}\left[\phi R^{(2)}(g)+V(\phi)\right],
$$

where $g_{\mu \nu}$ and $R^{(2)}$ are the two-dimensional, Weyl-rescaled, metric and Ricci scalar, respectively. Different choices of $V(\phi)$ identify different theories. Some remarkable examples are the Callan-Giddings-Harvey-Strominger (CGHS) model $[15](V=$ const $)$, the Jackiw-Teitelboim model [16] $(V=\phi)$, and the dimensionally reduced theory of four-dimensional spherically symmetric Einstein gravity integrated on a twosphere of area $16 \pi \phi[V=1 /(2 \sqrt{\phi})][2,17]$.

\footnotetext{
*Email address: cavaglia@aei-potsdam.mpg.de
}

According to their integrability properties, dilaton gravity models can be roughly divided into three classes.

(i) Completely integrable models, i.e., models that can be expressed in terms of free fields by a canonical transformation. Some remarkable examples are matterless dilaton gravity with an arbitrary potential [3] and the CGHS model [5,6].

(ii) Completely solvable models, i.e., models that cannot be analytically solved in terms of free fields but whose general solution is known. The two-dimensional effective generalized theory of $2+1$ cylindrical gravity minimally coupled to a massless scalar field [8] and dilaton gravity with constant or linear dilatonic potential minimally coupled to massless Dirac fermions $[9,10]$ belong to this class.

(iii) Partially integrable models, i.e., models that are integrable in a $(0+1)$-dimensional sector only, namely, after reduction to a finite number of degrees of freedom. In this category we find, for example, dilaton gravity minimally coupled to massless Dirac fermions with arbitrary potential [9] and two-dimensional effective models describing uncharged black $p$-branes in $N$ dimensions [13].

Completely integrable models are of particular interest from the quantum point of view. In this case we are able to quantize the theory (in the free-field representation) and, hopefully, to discuss quantization subtleties and nonperturbative quantum effects. (See, e.g., Refs. [5,6,18] for the CGHS model.) In particular, matterless dilaton gravity-Eq. (2) - can be used to describe black holes and, in the case of coupling with scalar matter, gravitational collapse. So the quantization program is worth exploring.

Although the classical properties of the model based upon Eq. (2) are well known, a conclusive word about its quantization is not known [2,19,20], even in the simplest (CGHS) case. Let us recall the two most fruitful attempts to construct a quantum theory of the CGHS model that are described in Refs. [5,6,18] and Ref. [21], respectively.

The first approach is based on a canonical transformation mapping the original system to a system described by freefields. Then the theory is quantized in the free-field representation. The main drawback of this approach is that the new canonical variables are not directly related to the original spacetime geometry and important physical quantities cannot be expressed in terms of the new fields [18]. Further, it is not clear how to generalize the canonical transformation for an arbitrary dilatonic potential. (Recently, a proof of the existence of a canonical transformation that generalizes the ca- 
nonical transformation used in the CGHS case has been derived by Cruz and Navarro-Salas; see Ref. [22]. Even though it seems reasonable to guess the existence of a canonical transformation in the general case, the relation between the new fields and the original geometrical variables remains a puzzle.)

The "geometrodynamical approach" was originally developed by Kuchar for a canonical description of the Schwarzschild black hole [23]. This approach uses variables that are directly related to the spacetime geometry and does not make use of the field redefinitions of Refs. [5,6,18]. Again, only the CGHS model has been quantized using this formalism [21].

In this paper we assume a different attitude and quantize the general matterless dilaton gravity model described by Eq. (2) using a transformation of the configuration space performed at the Lagrangian level. The transformation is suggested by the topological nature of two-dimensional gravity and by the existence of a local integral of motion independent of the coordinates first discussed by Filippov [3]. The new fields have clear physical meaning-they are the dilaton and the "mass" of the system-thus avoiding problems related to their interpretation in terms of the geometrical variables.

In the canonical framework the new fields generalize the geometrodynamical variables of Kuchar [23] and Varadarajan [21] to a generic dilatonic potential. Thus the quantization is straightforward and can be completed along the lines of Refs. [23,21]. The quantum theory reduces to quantum mechanics and the Hilbert space coincides with the Hilbert space obtained by quantizing the theory first reducing it to a $0+1$ dynamical system with a finite number of degrees of freedom and then imposing the quantization algorithm [see Ref. [27] for the case $V=1 /(2 \sqrt{\phi})]$. This result represents the quantum generalization of the well-known Birkhoff theorem $[24,25]$ for spherically symmetric gravity in four dimensions. (A somewhat different derivation of the so-called quantum Birkhoff theorem for the CGHS model is discussed in Ref. [18]. The approach of Ref. [18] makes use of the canonical transformation to free fields. Here we extend the results of Ref. [18] to the general model with an arbitrary dilatonic potential using a different and more powerful approach.)

The quantum Birkhoff theorem is schematically defined by the following diagram:

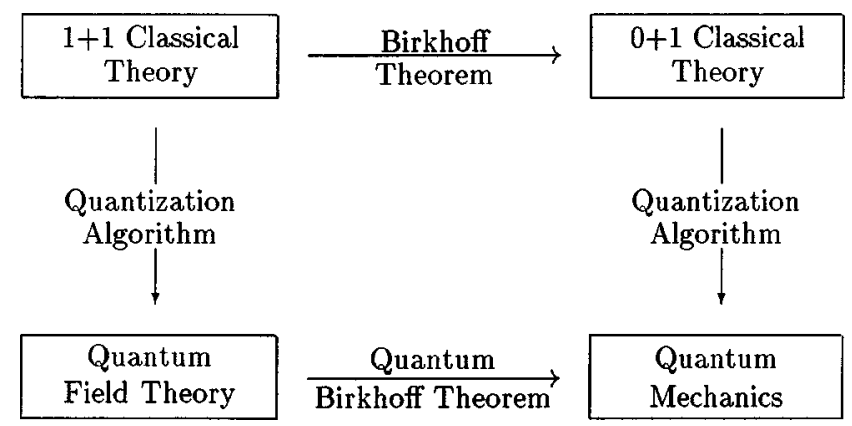

The outline of the paper is as follows. In the next section we present the classical theory of two-dimensional dilaton gravity (see Refs. $[2,19,26,25])$ following the approach developed by Filippov [3]. Although this section reviews essentially previous work, its content is useful to set up the notation and make the paper more self-contained and readable. In Sec. III we introduce the point transformation and the new Lagrangian. This result follows from the integrability properties of the system and constitutes the main contribution of the paper. Indeed, the Lagrangian formulation is at the basis of the canonical formalism and leads straightforwardly to the geometrodynamical variables. The canonical framework is discussed in Sec. IV. First, we derive the canonical transformation to the geometrodynamical variables for the general model. Then we present a careful treatment of falloff conditions which are essential in establishing the Hamiltonian quantization. The discussion of falloff conditions involves subtleties related to the definition of boundary conditions for arbitrary spacetimes. In Sec. V we quantize the system. Thanks to the geometrodynamical variables the quantization of the general model can be achieved by implementing the formalism developed by Kuchar for the quantization of the Schwarzschild black hole [23]. Finally, we show that the ensuing quantum theory is equivalent to the quantum mechanical theory which is obtained by imposing the Birkhoff theorem at the classical level. In Sec. VI we state our conclusions.

\section{CLASSICAL THEORY}

Let us consider Eq. (2). Varying the action with respect to the metric and the dilaton we obtain [25]

$$
\begin{gathered}
\left(\nabla_{(\mu} \nabla_{\nu)}-g_{\mu \nu} \nabla_{\sigma} \nabla^{\sigma}\right) \phi+\frac{1}{2} g_{\mu \nu} V(\phi)=0, \\
R+\frac{d V}{d \phi}=0
\end{gathered}
$$

where the symbol $\nabla$ represents covariant derivatives with respect to the metric $g_{\mu \nu}$.

It is easy to prove that Eq. (5) is satisfied if Eq. (4) is satisfied provided that

$$
H\left(g_{\mu \nu}, \phi\right) \neq 0,
$$

where $H\left(g_{\mu \nu}, \phi\right)=\nabla_{\rho} \phi \nabla^{\rho} \phi$. This condition can be lifted if one requires the continuity of the fields and of their derivatives at any spacetime point. We will see in a moment-see Eq. (17) below-that the equation $H\left(g_{\mu \nu}, \phi\right)=0$ defines the horizon(s) of the two-dimensional metric. So by requiring the continuity of the fields and their derivatives across the horizon(s) Eq. (4) implies Eq. (5) everywhere.

The field equations (4),(5) can be solved performing a Bäcklund transformation (see Ref. [3]). In covariant language the Bäcklund transformation reads

$$
M=N(\phi)-\nabla_{\rho} \phi \nabla^{\rho} \phi, N(\phi)=\int^{\phi} d \phi^{\prime} V\left(\phi^{\prime}\right),
$$




$$
\nabla_{\mu} \psi=\frac{\nabla_{\mu} \phi}{\nabla_{\rho} \phi \nabla^{\rho} \phi},
$$

where $M(t, x)$ and $\psi(t, x)$ are the transformed fields. ( $M$ and $N$ coincides-apart from constant factors - to $C$ and $J$ of Refs. [19,2] respectively.) Note that the transformation is singular for $H\left(g_{\mu \nu}, \phi\right)=0$. Using the new fields Eq. (4) reads

$$
\begin{gathered}
\nabla_{\mu} \nabla^{\mu} \psi=0, \\
\nabla_{\mu} M=0 .
\end{gathered}
$$

Since the transformation (7),(8) is defined when Eq. (6) holds, Eqs. (9),(10) are equivalent to the original field equations (4),(5) except at the horizon(s). Equations (9),(10) have a deep significance. The first equation implies that $\psi$ is a free (D'Alembert) field. From the second equation we find that $M$ is a locally conserved quantity.

In two dimensions any metric is locally conformally flat [28]. So there exists a coordinate transformation which brings the metric into the form

$$
d s^{2}=4 \rho(u, v) d u d v,
$$

where $u=(t+x) / 2, v=(t-x) / 2$. Using conformal lightcone coordinates Eqs. (9),(10) can be explicitly integrated. The general solution is

$$
\psi=U(u)+V(v), \quad M=M_{0} .
$$

The original fields $\rho$ and $\phi$ are can be written as functions of $\psi$ and $M$ using Eqs. (7),(8). With a little algebra one finds

$$
\begin{gathered}
\frac{d \psi}{d \phi}=\frac{1}{N(\phi)-M}, \\
\rho=[N(\phi)-M] \partial_{u} \psi \partial_{v} \psi .
\end{gathered}
$$

Equations (12)-(14) imply that the general solution of the model is actually $(0+1)$ dimensional, i.e., that any solution possesses a Killing vector [29]. Indeed, using the coordinates $(U, V)$ the general solution reads

$$
d s^{2}=4[N(\phi)-M] d U d V, \quad \phi \equiv \phi(U+V),
$$

or, using the coordinates $(\phi, T \equiv U-V)$,

$$
d s^{2}=-[N(\phi)-M] d T^{2}+[N(\phi)-M]^{-1} d \phi^{2} .
$$

Thus the general solution depends on the single variable $\phi$. (With a somewhat improper terminology we call these solutions static, even though the Killing vector may not be timelike and hypersurface orthogonal on the entire manifold.) This result constitutes a generalization of the classical Birkhoff theorem [25,24,3,29]. (For spherically symmetric Einstein gravity the "local integral of motion independent of the coordinates"' is just the Schwarzschild mass.) The reduction of the theory to a finite-dimensional dynamical system signals that pure dilaton gravity is actually a topological theory. In Sec. V we will see how this property influences the quantization of the theory.

Let us briefly discuss the local geometrical properties of the solution (16). The horizon(s) of the metric are determined by the equation

$$
N(\phi)-M \equiv H\left(g_{\mu \nu}, \phi\right)=0 .
$$

For a given choice of the dilatonic potential, Eq. (17) is an algebraic equation in $\phi$ whose solutions $\left\{\phi_{i}\right\}$ determine the values of the radial coordinate where the horizon(s) are located. So the request of continuity of the solution-and of its derivatives (see Ref. [23]) — across the horizons enforces the continuity of the fields $\rho$ and $\phi$ at the points $H\left(g_{\mu \nu}, \phi\right)$ $=0$ and vice versa. This justifies a posteriori the assumption of continuity made below Eq. (6). With this assumption Eqs. (4),(5) are equivalent to Eqs. (9),(10) everywhere.

The local asymptotic structure of the solution (16) and the existence of singularities depend on the choice of the dilatonic potential. In particular, from Eq. (5) one finds that singularities of the metric are determined by singular points of the first derivative of $V(\phi)$ with respect to $\phi$. The local asymptotic structure can be also roughly investigated using Eq. (5). For instance, let us suppose that the asymptotic region is defined by $\phi \rightarrow \infty$ and that the behavior of the dilatonic potential at infinity is $V(\phi) \approx \phi^{k}$, where $k$ is a constant parameter. Thus the two-dimensional spacetime is asymptotically flat for $\phi \rightarrow \infty$ if $k<1$, and has constant curvature for $\phi \rightarrow \infty$ if $k=1$.

Let us conclude this section with a concrete example and derive the Schwarzschild solution using the formalism described above. The dimensional reduction of the fourdimensional vacuum Einstein gravity,

$$
S_{E H}=\frac{1}{16 \pi} \int_{\Sigma} d^{4} x \sqrt{-g} R^{(4)}(g),
$$

can be obtained using the ansatz

$$
d s_{(4)}^{2}=\frac{1}{\sqrt{\phi}} g_{\mu \nu} d x^{\mu} d x^{\nu}+4 \phi d \Omega^{2}, \quad \phi \geqslant 0
$$

where $g_{\mu \nu}$ is a two-dimensional metric with signature $(-1,1)$ and $d \Omega^{2}$ is the line element of the unit two-sphere. Using Eq. (19), and integrating on the two-sphere, the fourdimensional Einstein-Hilbert action can be cast into the form (2) with $V(\phi)=1 /(2 \sqrt{\phi})$. Using Eq. (16) the line element (19) reads

$$
d s_{(4)}^{2}=-\left(1-\frac{M}{\sqrt{\phi}}\right) d T^{2}+\frac{d \phi^{2}}{\phi\left(1-\frac{M}{\sqrt{\phi}}\right)}+4 \phi d \Omega_{2}^{2} \text {. }
$$

Clearly Eq. (20) reduces to the standard Schwarzschild solution with the substitution $4 \phi=R^{2}$. 


\section{LAGRANGIAN FORMALISM}

The Bäcklund transformation introduced in the previous section can be used to find a transformation from the original fields, $\left(g_{\mu \nu}, \phi\right)$, to new fields $\left(X_{i}\right), i=1, \ldots, 4$, where one of the new fields $X_{i}$ coincides with $M$. Since $M$ is a locally conserved quantity, this transformation simplifies drastically the dilaton gravity Lagrangian in Eq. (2).

The key of the construction is the observation that in two dimensions the Ricci scalar $R$ is a total divergence and can be locally written as

$$
\frac{R}{2}=\nabla_{\mu} A^{\mu}, \quad A^{\mu}=\frac{\nabla^{\mu} \nabla^{\nu} \chi \nabla_{\nu} \chi-\nabla_{\nu} \nabla^{\nu} \chi \nabla^{\mu} \chi}{\nabla_{\rho} \chi \nabla^{\rho} \chi},
$$

where $\chi$ is an arbitrary, nonconstant, function of the coordinates. Equation (21) can be easily checked using conformal coordinates. Since Eq. (21) is a generally covariant expression, and any two-dimensional metric can be locally cast in the form (11) by a coordinate transformation [28], Eq. (21) is valid in any system of coordinates.

Differentiating Eq. (7), and choosing $\chi=\phi$, both $V(\phi)$ and $R$ can be written as functions of $M$ and $\nabla_{\mu} \phi$. Finally, by an integration per parts we find

$$
S=\int_{\Sigma} d^{2} x \sqrt{-g} \frac{\nabla_{\mu} \phi \nabla^{\mu} M}{N(\phi)-M}+S_{\partial},
$$

where $S_{\partial}$ is the surface term:

$$
S_{\partial}=2 \int_{\Sigma} d^{2} x \sqrt{-g} \nabla_{\mu}\left[\nabla^{\mu} \phi+\phi A^{\mu}\right] .
$$

Let us check that Eq. (22) has the same number of degrees of freedom (DOF) of the original action (2). In two dimensions a generic metric can be written

$$
g_{\mu \nu}=\rho\left(\begin{array}{cc}
\alpha^{2}-\beta^{2} & \beta \\
\beta & -1
\end{array}\right) .
$$

In the canonical formalism $\alpha(t, x)$ and $\beta(t, x)$ play the role of the lapse function and of the shift vector respectively; $\rho(t, x)$ is the dynamical DOF. As a result of the chosen parametrization, the Lagrangian in Eq. (2) is a functional of the two dynamical fields $(\rho, \phi)$ and of the two nondynamical variables $(\alpha, \beta)$. Now let us use Eq. (24) in Eq. (22) and neglect the surface term. The new Lagrangian is again a functional of two fields $(M, \phi)$ and of two nondynamical variables $(\alpha, \beta)$. Indeed, since Eq. (22) only contains the Weyl-invariant combinations $\sqrt{-g} g^{\mu \nu}$, the transformed action is invariant under changes of coordinates which belong to the conformal group and $g_{\mu \nu}$ does not contribute any dynamical DOF to the action. As a consequence, the transformation $(\rho, \phi, \alpha, \beta) \rightarrow(M, \phi, \alpha, \beta)$ is a "point transformation" with $M \equiv M(\rho, \phi, \alpha, \beta)$ defined by Eq. (7). [Quotation marks are due to the fact that the transformation $(\rho, \phi, \alpha, \beta) \rightarrow(M, \phi, \alpha, \beta)$ should not be regarded as a point transformation according to the usual lore because it in- volves derivatives with respect to $t$ and $x$. We call it a "point transformation", because it can be implemented at the Lagrangian level.]

Varying Eq. (22) we find

$$
\begin{gathered}
\nabla_{\mu} \nabla^{\mu} \phi-V(\phi)=0, \\
\nabla_{(\mu} \phi \nabla_{\nu)} M-\frac{1}{2} g_{\mu \nu} \nabla_{\sigma} \phi \nabla^{\sigma} M=0, \\
\nabla_{\mu} M \nabla^{\mu} M+\nabla_{\nu} \phi \nabla^{\nu} \phi \nabla_{\mu} \nabla^{\mu} M=0 .
\end{gathered}
$$

Equations (25)-(27) are equivalent to the field equations obtained from Eq. (2). Equation (25) corresponds to the trace of Eq. (4). Further, by differentiation of Eq. (7) one finds that Eqs. (26),(27) are satisfied if Eq. (4) is satisfied because Eq. (4) implies $\nabla_{\mu} M=0$. The converse latter statement is also true provided that Eq. (6) is satisfied. When this condition holds Eq. (26) implies $\nabla_{\mu} M=0$. By requiring the continuity of the fields Eqs. (25)-(27) and Eqs. (4),(5) are equivalent. The equivalence of Eqs. (25)-(27) and the original field equations can also be directly checked using the metric parametrization defined in Eq. (24).

\section{CANONICAL FORMALISM}

The canonical formalism is an essential step in the quantization procedure. Starting from Eq. (2), and using the metric parametrization, Eq. (24), the action can be cast in the Hamiltonian form [2]

$$
S=\int d t \int_{x_{a}}^{x_{b}} d x\left[\pi_{\rho} \dot{\rho}+\pi_{\phi} \dot{\phi}-\alpha \mathcal{H}_{0}-\beta \mathcal{H}_{1}\right],
$$

where the overdots represent derivatives with respect to the timelike coordinate $t,\left(\rho, \phi, \pi_{\rho}, \pi_{\phi}\right)$ are the phase space variables, and $\mathcal{H}_{0}, \mathcal{H}_{1}$ are the Arnowitt-Deser-Misner (ADM) super-Hamiltonian and supermomentum, respectively:

$$
\begin{gathered}
\mathcal{H}_{0}=\rho \pi_{\rho} \pi_{\phi}+\frac{\rho^{\prime}}{\rho} \phi^{\prime}-2 \phi^{\prime \prime}-\rho V(\phi), \\
\mathcal{H}_{1}=-\phi^{\prime} \pi_{\phi}+\rho^{\prime} \pi_{\rho}+2 \rho \pi_{\rho}^{\prime} .
\end{gathered}
$$

Here primes represent derivatives with respect to the spatial coordinate $x$. Equations (29) include, as particular cases, the models discussed in Ref. [21] and Ref. [23]. Denoting with subscripts $v$ and $k$ the canonical variables of Ref. [21] and [23], respectively, we have

$$
\begin{gathered}
\phi=\frac{R_{v}^{2}}{4}, \pi_{\phi}=2 \frac{R_{v} P_{R_{v}}-\Lambda_{v} P_{\Lambda_{v}}}{R_{v}^{2}}, \\
\rho=R_{v}^{2} \Lambda_{v}^{2}, \quad \pi_{\rho}=\frac{P_{\Lambda_{v}}}{2 R_{v}^{2} \Lambda_{v}},
\end{gathered}
$$




$$
\alpha=-\frac{N_{v}}{\Lambda_{v}}, \beta=-N_{v}^{r},
$$

for the CGHS model ( $V=$ const), and

$$
\begin{gathered}
\phi=\frac{R_{k}^{2}}{4}, \quad \pi_{\phi}=\frac{2 R_{k} P_{R_{k}}-\Lambda_{k} P_{\Lambda_{k}}}{R_{k}^{2}}, \\
\rho=\frac{R_{k} \Lambda_{k}^{2}}{2}, \quad \pi_{\rho}=\frac{P_{\Lambda_{k}}}{R_{k} \Lambda_{k}}, \\
\alpha=-\frac{N_{k}}{\Lambda_{k}}, \quad \beta=-N_{k}^{r},
\end{gathered}
$$

for the Schwarzschild black hole $V=-1 /(2 \sqrt{\phi})$. [The minus sign of $V$ is due to the choice of the metric signature in Eq. (24) that is opposite to the signature used in Eq. (19).]

Starting from Eq. (22) the super-Hamiltonian and supermomentum read (for later convenience we set $\phi=\bar{\phi}$ )

$$
\begin{gathered}
\mathcal{H}_{0}=[N(\bar{\phi})-M] \pi_{\bar{\phi}} \pi_{M}+[N(\bar{\phi})-M]^{-1} \bar{\phi}^{\prime} M^{\prime}, \\
\mathcal{H}_{1}=-\bar{\phi}^{\prime} \pi_{\bar{\phi}}-M^{\prime} \pi_{M} .
\end{gathered}
$$

Eventually, both canonical actions must be complemented by a boundary term at the spatial boundaries. This can be done along the lines of Refs. $[23,21]$ as we will see later in this section.

The two charts $\left(\phi, \pi_{\phi}, \rho, \pi_{\rho}\right)$ and $\left(\bar{\phi}, \pi_{\bar{\phi}}, M, \pi_{M}\right)$ are related by the transformation

$$
\begin{gathered}
M=N(\phi)-\frac{\rho^{2} \pi_{\rho}^{2}-\phi^{\prime 2}}{\rho}, \\
\pi_{M}=\frac{\rho^{2} \pi_{\rho}}{\rho^{2} \pi_{\rho}^{2}-\phi^{\prime 2}}, \\
\bar{\phi}=\phi \\
\pi_{\bar{\phi}}=\pi_{\phi}-\frac{\rho^{2} \pi_{\rho}}{\rho^{2} \pi_{\rho}^{2}-\phi^{\prime 2}}\left[V(\phi)+2 \pi_{\rho}\left(\frac{\phi^{\prime}}{\rho \pi_{\rho}}\right)^{\prime}\right] .
\end{gathered}
$$

The transformation given above is easily invertible. The result is

$$
\begin{gathered}
\rho=\pi_{M}^{2}[N(\bar{\phi})-M]\left[1-\left(\frac{\bar{\phi}^{\prime}}{\pi_{M}[N(\bar{\phi})-M]}\right)^{2}\right], \\
\pi_{\rho}=\frac{1}{\pi_{M}\left[1-\left(\frac{\bar{\phi}^{\prime}}{\pi_{M}[N(\bar{\phi})-M]}\right)^{2}\right]}, \\
\phi=\bar{\phi},
\end{gathered}
$$

$$
\pi_{\phi}=\pi_{\bar{\phi}}+V(\bar{\phi}) \pi_{M}+\frac{2\left(\frac{\bar{\phi}^{\prime}}{\pi_{M}[N(\bar{\phi})-M]}\right)^{\prime}}{\left[1-\left(\frac{\bar{\phi}^{\prime}}{\pi_{M}[N(\bar{\phi})-M]}\right)^{2}\right]}
$$

After some tedious calculations one can check that the only nonvanishing Poisson brackets at equal time $t$ are

$$
\begin{aligned}
& {\left[M(t, x), \pi_{M}\left(t, x^{\prime}\right)\right]=\delta\left(x-x^{\prime}\right),} \\
& {\left[\bar{\phi}(t, x), \pi_{\bar{\phi}}\left(t, x^{\prime}\right)\right]=\delta\left(x-x^{\prime}\right) ;}
\end{aligned}
$$

so Eqs. (33),(34) define a canonical map. Finally, the difference of the Liouville forms reads

$$
\begin{aligned}
& \int_{x_{a}}^{x_{b}} d x\left(\dot{M} \pi_{M}+\dot{\bar{\phi}} \pi_{\bar{\phi}}\right)-\int_{x_{a}}^{x_{b}} d x\left(\dot{\rho} \pi_{\rho}+\dot{\phi} \pi_{\phi}\right) \\
& \quad=F\left(\bar{\phi}, \pi_{\bar{\phi}}, M, \pi_{M}\right),
\end{aligned}
$$

where

$$
\begin{aligned}
& F\left(\bar{\phi}, \pi_{\bar{\phi}}, M, \pi_{M}\right) \\
& =\int_{x_{a}}^{x_{b}} d x\left\{2 \bar{\phi}^{\prime} \operatorname{arctanh}\left[\frac{\bar{\phi}^{\prime}}{[N(\bar{\phi})-M] \pi_{M}}\right]\right. \\
& \left.-2(N(\bar{\phi})-M) \pi_{M}\right\}^{\cdot} \\
& \quad-\int_{x_{a}}^{x_{b}} d x\left\{2 \dot{\bar{\phi}} \operatorname{arctanh}\left[\frac{\bar{\phi}^{\prime}}{[N(\bar{\phi})-M] \pi_{M}}\right]\right\}^{\prime}
\end{aligned}
$$

The canonical variables $\left(\bar{\phi}, \pi_{\bar{\phi}}, M, \pi_{M}\right)$ are a generalization of the geometrodynamical variables introduced by $\mathrm{Ku}-$ char [23] and Varadarajan [21]. This can be easily proved using Eqs. (30),(31) and Eqs. (33),(34).

Now we must take care of boundary terms and define falloff conditions at the spatial boundaries. We set

$$
\begin{gathered}
\bar{\phi}^{\prime}=[N(\bar{\phi})-M]\left(1+\epsilon_{\phi^{\prime}}^{-(a, b)}\right), \\
\dot{\bar{\phi}}=\epsilon_{\dot{\bar{\phi}}}^{(a, b)}, \\
M=M^{(a, b)}(t)\left(1+\epsilon_{M}^{(a, b)}\right), \\
\pi_{\bar{\phi}}^{-}=\frac{\epsilon_{\pi_{\bar{\phi}}}^{(a, b)}}{N(\bar{\phi})-M}, \\
\pi_{M}=\frac{\epsilon_{\pi_{M}}^{(a, b)}}{N(\bar{\phi})-M}, \\
\alpha=\alpha^{(a, b)}(t)\left(1+\epsilon_{\alpha}^{(a, b)}\right),
\end{gathered}
$$




$$
\beta=\epsilon_{\beta}^{(a, b)},
$$

where $\epsilon^{(a, b)}$ are functions of $t$ and $x$ vanishing at the spatial boundaries $x_{a}$ and $x_{b}$, i.e.,

$$
\lim _{x \rightarrow x_{a}, x_{b}} \epsilon^{(a, b)}(t, x)=0
$$

and

$$
\begin{aligned}
& \lim _{x \rightarrow x_{a}, x_{b}} \frac{\epsilon_{\pi_{\bar{\phi}}}^{(a, b)}}{N(\bar{\phi})-M}=0, \\
& \lim _{x \rightarrow x_{a}, x_{b}} \frac{\epsilon_{\pi_{M}}^{(a, b)}}{N(\bar{\phi})-M}=0, \\
& \lim _{x \rightarrow x_{a}, x_{b}} \frac{\epsilon_{M}^{(a, b)^{\prime}}}{N(\bar{\phi})-M}=0 .
\end{aligned}
$$

The exact behavior of the $\epsilon^{(a, b)}$ functions depends on the particular potential under consideration. By requiring that $\epsilon^{(a, b)}$ go to zero rapidly enough, both the Liouville form and the super-Hamiltonian and supermomentum are well defined and the difference of the Liouville forms $F\left(\bar{\phi}, \pi_{\bar{\phi}}, M, \pi_{M}\right)$ reduces to an exact form. For instance, in the Schwarzschild black hole case $N(\phi)=\sqrt{\phi}$ the spatial boundaries are located at $x_{a}=-\infty$ and $x_{b}=+\infty$ and with a little algebra one can check that the falloff conditions (38) become

$$
\begin{gathered}
\bar{\phi}=\frac{x^{2}}{4}\left[1+\bar{\phi}_{ \pm}|x|^{-1-\epsilon}+O^{ \pm \infty}\left(|x|^{-2-\epsilon}\right)\right], \\
M=M_{ \pm}(t)\left[1+O^{ \pm \infty}\left(|x|^{-\epsilon}\right)\right], \\
\pi_{\bar{\phi}}=O^{ \pm \infty}\left(|x|^{-2-\epsilon}\right), \\
\pi_{M}=O^{ \pm \infty}\left(|x|^{-1-\epsilon}\right), \\
\alpha=\alpha_{ \pm}(t)\left[1+O^{ \pm \infty}\left(|x|^{-\epsilon}\right)\right], \\
\beta=O^{ \pm \infty}\left(|x|^{-\epsilon}\right), 0<\epsilon \leqslant 1 .
\end{gathered}
$$

In this case the super-Hamiltonian and supermomentum fall off as

$$
\mathcal{H}_{0}=O^{ \pm \infty}\left(|x|^{-1-\epsilon}\right), \quad \mathcal{H}_{1}=O^{ \pm \infty}\left(|x|^{-1-\epsilon}\right),
$$

and the Liouville form

$$
\int_{-\infty}^{+\infty} d x\left(\dot{M} \pi_{M}+\dot{\bar{\phi}} \pi_{\bar{\phi}}\right), \quad \dot{M} \pi_{M}+\dot{\bar{\phi}} \pi_{\bar{\phi}}=O^{ \pm \infty}\left(|x|^{-1-\epsilon}\right)
$$

is well defined. Finally, using Eqs. (41) the second term on the right-hand side of Eq. (37) vanishes at spatial infinities and the difference of the Liouville forms is an exact form. The above falloff conditions coincide with those used by Kuchař in Ref. [23].
Following [30] we must complement the action by a boundary term to allow functional differentiability of the action. Using the falloff conditions (38) we have that the sole nonvanishing boundary term due to the variation of the action with respect to the canonical variables has the form

$$
\int d t\left[-\alpha^{(b)}(t) \delta M^{(b)}+\alpha^{(a)}(t) \delta M^{(a)}\right]
$$

so we add to the action the boundary term

$$
S_{\text {boundary }}=\int d t\left[\alpha^{(b)}(t) M^{(b)}(t)-\alpha^{(a)}(t) M^{(a)}(t)\right],
$$

where $\alpha^{(a, b)}(t)$ parametrize the action at the boundary and are interpreted as prescribed values of $t$ (see Ref. [23] for a more detailed discussion about this point).

The canonical field equations and the constraints $\mathcal{H}_{0}$ $=0, \mathcal{H}_{1}=0$ are easily solved using the geometrodynamical chart $\left(\bar{\phi}, \pi_{\bar{\phi}}, M, \pi_{M}\right)$. The general solution of the constraints is

$$
\pi_{\bar{\phi}}=0, M^{\prime}=0 \text {. }
$$

Equations (46) have the same physical content of Eq. (10). (Note that $M$ weakly commutes with the constraints, as expected for a local integral of motion.) Equation (9) is a direct consequence of the canonical field equations.

\section{QUANTIZATION}

The quantization of the full $1+1$ theory can be implemented using the geometrodynamical canonical variables. From Eqs. (46) we read that $M$ does not depend on the spacelike coordinate $x$. The effective Hamiltonian is simply given by the boundary term (45) and the reduced action reads

$$
S_{\mathrm{eff}}=\int d \tau\left[\frac{d m}{d \tau} p_{m}-m\right],
$$

where $m \equiv M^{(b)}(t)=M^{(a)}(t), \quad p_{m} \equiv \int_{x_{a}}^{x_{b}} d x \pi_{M}$, and $\tau(t)$ $=\int{ }^{t} d t^{\prime}\left[\alpha^{(a)}\left(t^{\prime}\right)-\alpha^{(b)}\left(t^{\prime}\right)\right]$. The theory reduces formally to quantum mechanics and the quantization can be carried on as usual. The Schrödinger equation is

$$
i \frac{\partial}{\partial \tau} \Psi(m ; \tau)=\mathcal{H}_{\mathrm{eff}} \Psi(m ; \tau), \quad \mathcal{H}_{\mathrm{eff}} \equiv m
$$

The stationary states are the eigenfunctions of $m$ and the Hilbert space coincides with the Hilbert space obtained in the $0+1$ approach. Let us see briefly this point in detail.

In the $0+1$ approach we take advantage that every solution is static-according to the definition given below Eq. (16)—and set from the beginning $g_{\mu \nu} \equiv g_{\mu \nu}(t), \phi \equiv \phi(t)$. The action (density) reads

$$
S_{0+1}=\int d t\left[\dot{\rho} \pi_{\rho}+\dot{\phi} \pi_{\phi}-\alpha \mathcal{H}\right]
$$


where $\alpha$ is a Lagrange multiplier enforcing the constraint $\mathcal{H}=0$. ( $\mathcal{H}$ corresponds to the $0+1$ slice of $\mathcal{H}_{0}$. The supermomentum constraint $\mathcal{H}_{1}$ vanishes identically.)

So in the $0+1$ sector of the theory we can express the field equations as a canonical system in a finite, $(2 \times 2)$ dimensional, phase space. The equations of motion are analytically integrable and their solution coincides with the finite gauge transformation generated by the constraint $\mathcal{H}$ $=0$. We can find a couple of gauge-invariant canonically conjugate quantities $m$ and $p_{m}$ corresponding to the $0+1$ sections of $M$ and $\pi_{M}$ introduced in Eqs. (33). The canonical variables $m$ and $p_{m}$ can be identified with the quantities defined below Eq. (47).

Now we can construct the maximal gauge-invariant canonical chart $\left(m, p_{m}, \mathcal{H}, \mathcal{T}\right)$ and use $\mathcal{T}$ to fix the gauge. Indeed, the transformation properties of $\mathcal{T}$ under the gauge transformation generated by $\mathcal{H}$ imply that time defined by this variable covers once and only once the symplectic manifold; i.e., time defined by $\mathcal{T}$ is a global time. The quantization becomes trivial and the Hilbert space is spanned by the eigenvectors of the sole-apart from its conjugate momentum-gauge-invariant operator $m$ corresponding to the mass of the system.

This quantization program has been implemented in detail in Ref. [27] for the case of spherically symmetric Einstein gravity but can be easily generalized to an arbitrary $V(\phi)$. (See, for instance, Ref. [31].) In the $0+1$ approach one can go further and discuss the self-adjointness properties of the mass operator. It turns out that the Hermitian operator $m$ in the gauge-fixed, positive-norm, Hilbert space is not selfadjoint, while its square is a self-adjoint operator with positive eigenvalues. This result is due to the fact that the conjugate variable to the mass, $p_{m}$, has positive support, analogously to what happens for the radial momentum in ordinary quantum mechanics. However, the relevant point is that the mass $m$-or its square-is the only gauge-invariant observable of the system (apart from the conjugate variable, of course) and the Hilbert space of the $0+1$ approach coincides with the Hilbert space of the full quantum $1+1$ theory obtained through the geometrodynamical formalism. This is the essence of the quantum Birkhoff theorem. Note that our definition of the quantum Birkhoff theorem differs from the definition that can be found in the previous literature $[2,19]$. (Actually, in the previous literature a precise definition of the quantum Birkhoff theorem is missing.) We define the Birkhoff theorem as the equivalence of the $(0+1)$ - and $(1$ $+1)$-dimensional quantization procedures-see the diagram at the end of Sec. I. This definition gives a clear meaning to the quantum Birkhoff theorem that up to now was simply intended as the property that quantum states depend on a single parameter (the mass of the system). While conclusions are identical, conceptual differences are great.

\section{CONCLUSIONS AND PERSPECTIVES}

Let us conclude with few remarks. We have derived a canonical transformation to geometrodynamical variables that generalizes the transformation of Ref. [21] and Ref. [23] to any dilaton gravity model. We have seen that the general dilaton gravity action, Eq. (2), can be cast into the form (22) and the system can be described both in the canonical and Lagrangian frameworks using the dilaton and the mass as new variables. The quantization of the general dilaton gravity model becomes straightforward and the resulting quantum theory exhibits at the quantum level the Birkhoff theorem.

We believe that Eq. (22) and Eqs. (32) can be used to look at two-dimensional gravity from a new perspective. Up to now physicists have struggled themselves to find a canonical transformation mapping the general dilaton gravity theory based upon Eq. (2) into a system described by free fieldssee for instance, Ref. [22]. Even though it seems reasonable to assume the existence of such a canonical transformation, we know from the CGHS case (the simplest possible case) that the relation between the free fields and the "physical" fields (metric, dilaton, mass) is highly nonlinear. Further, the canonical transformation may be pathological and subtleties and ambiguities may arise. For instance, in the CGHS case the gauge-invariant operator $M$ cannot be expressed as a function of the new free fields, as we would expect implementing a canonical transformation [18]. This means that the canonical transformation to free fields is ill defined. Indeed, a careful analysis shows that the transformation cannot be inverted and, in order to make it invertible, one has to supplement the new field variables by an extra pair of conjugate variables related to the value of the fields at the boundary. (See, for instance, [18] and [6].) In spite of these difficulties, the CGHS model can still be managed and different approaches to the quantization can be carried on, leading to a consistent quantum theory $[5,6,18]$. However, we find it very hard to believe that models with more complicated dilatonic potentials can be dealt with using free fields. Eventually, one wants quantum operators corresponding to the physical quantities of the model, i.e., quoting Kuchar, Romano, and Varadarajan, " ... the interesting questions in dilatonic gravity are precisely those which are concerned with the physical spacetime..." [6]. Free fields are very distant from this picture.

Conversely, the canonical variables defined in Eq. (33), being directly related to the spacetime geometry, do not suffer from the problems outlined above. Thus a quantum theory in which quantum operators have a clear physical meaning is easily achieved. Finally, Eq. (22) may (hopefully) provide a completely new starting point in the investigation of open issues as, for instance, the thermodynamics of black holes and gravitational collapse (when matter is coupled to the system).

\section{ACKNOWLEDGMENTS}

I am indebted to Vittorio de Alfaro, Alexandre T. Filippov, Claus Kiefer, Jorma Louko, and Dmitri Vassilevich for many interesting discussions and useful suggestions about the subject of this paper. This work has been supported by a Human Capital and Mobility grant of the European Union, Contract No. ERBFMRX-CT96-0012. 
[1] An updated collection of papers on lower-dimensional gravity can be found at the web page http://www.aei-potsdam.mpg.de/ mc-cgi-bin/ldg.html.

[2] D. Louis-Martinez, J. Gegenberg, and G. Kunstatter, Phys. Lett. B 321, 193 (1994).

[3] A.T. Filippov, in "Problems in Theoretical Physics,' Dubna, JINR, 1996, p. 113; Mod. Phys. Lett. A 11, 1691 (1996); Int. J. Mod. Phys. A 12, 13 (1997).

[4] R. Jackiw, in Proceedings of the Second Meeting on Constrained Dynamics and Quantum Gravity [Nucl. Phys. B (Proc. Suppl.) 57, 162 (1997)]; M. Cavaglià, in Proceedings of the Sixth International Symposium on Particles, Strings and Cosmology (PASCOS-98) (World Scientific, Singapore, in press); M. Cavaglià, in Particles, Fields \& Gravitation, edited by J. Rembielinski, AIP Conf. Proc. No. 453 (AIP, Woodbury, NY, 1998), pp. 442-448.

[5] E. Benedict, R. Jackiw, and H.-J. Lee, Phys. Rev. D 54, 6213 (1996); D. Cangemi, R. Jackiw, and B. Zwiebach, Ann. Phys. (N.Y.) 245, 408 (1995).

[6] K.V. Kuchař, J.D. Romano, and M. Varadarajan, Phys. Rev. D 55, 795 (1997).

[7] T. Klösch, in Proceedings of the Second Meeting on Constrained Dynamics and Quantum Gravity [Nucl. Phys. B (Proc. Suppl.) 57, 326 (1997)]; T. Strobl, ibid, p. 330.

[8] M. Cavaglià, Phys. Rev. D 57, 5295 (1998).

[9] M. Cavaglià, L. Fatibene, and M. Francaviglia, Class. Quantum Grav. 15, 3627 (1998).

[10] H. Pelzer and T. Strobl, Class. Quantum Grav. 15, 3803 (1998).

[11] W. Kummer, H. Liebl, and D.V. Vassilevich, Nucl. Phys. B493, 491 (1997).

[12] W. Kummer, H. Liebl, and D.V. Vassilevich, Nucl. Phys. B513, 723 (1998).

[13] M. Cavaglià, Phys. Lett. B 413, 287 (1997).

[14] For a careful discussion about the role of conformal transformations in two-dimensional dilaton gravity see M.O. Katanaev, W. Kummer, and H. Liebl, Nucl. Phys. B486, 353 (1997) and Ref. [11].

[15] C. Callan, S. Giddings, J. Harvey, and A. Strominger, Phys.
Rev. D 45, 1005 (1992); H. Verlinde, in Sixth Marcel Grossmann Meeting on General Relativity, edited by M. Sato and T. Nakamura (World Scientific, Singapore, 1992); B.M. Barbashov, V.V. Nesterenko, and A.M. Chervjakov, Theor. Math. Phys. 40, 15 (1979).

[16] R. Jackiw and C. Teitelboim, in Quantum Theory of Gravity, edited by S. Christensen (Hilger, Bristol, 1984).

[17] J. Gegenberg and G. Kunstatter, Phys. Rev. D 47, R4192 (1993).

[18] M. Cavaglià, V. de Alfaro, and A.T. Filippov, Phys. Lett. B 424, 265 (1998); Atti Acad. Sci. Torino Sci. Fis. Mat. Natur. 131, 65 (1967).

[19] D. Louis-Martinez and G. Kunstatter, Phys. Rev. D 52, 3494 (1995).

[20] See, e.g., D. Louis-Martinez, Phys. Rev. D 55, 791 (1997); A. Barvinsky and G. Kunstatter, Phys. Lett. B 389, 231 (1996).

[21] M. Varadarajan, Phys. Rev. D 52, 7080 (1995).

[22] J. Cruz and J. Navarro-Salas, Mod. Phys. Lett. A 12, 2345 (1997).

[23] K.V. Kuchař, Phys. Rev. D 50, 3961 (1994).

[24] Y. Kiem, Phys. Lett. B 322, 323 (1994).

[25] D. Louis-Martinez and G. Kunstatter, Phys. Rev. D 49, 5227 (1994).

[26] J. Gegenberg, G. Kunstatter, and D. Louis-Martinez, Phys. Rev. D 51, 1781 (1995).

[27] M. Cavaglià, V. de Alfaro, and A.T. Filippov, Int. J. Mod. Phys. D 4, 661 (1995); 5, 227 (1996).

[28] L.P. Eisenhart, Riemannian Geometry (Princeton University Press, Princeton, NJ, 1964).

[29] T. Klösch and T. Strobl, Class. Quantum Grav. 13, 965 (1996); 13, 2395 (1996); 14, 1689 (1997).

[30] T. Regge and C. Teitelboim, Ann. Phys. (N.Y.) 174, 463 (1974); A. Hanson, T. Regge, and C. Teitelboim, Constrained Hamiltonian Systems (Accademia Nazionale dei Lincei, Roma, 1976).

[31] V. de Alfaro, "Quantization of the Schwarzschild Black Hole and Dilaton Models," in Proceedings of the XIth International Conference on Problems of Quantum Field Theory, Dubna, Russia, 1998, edited by B. M. Barbashov, G. V. Efimov, and A. V. Efremov (Dubna, Russia, 1999), pp. 14-24. 